\title{
Generalized viscosity approximation methods for nonexpansive mappings
}

Peichao Duan* and Songnian He

"Correspondence:

pcduancauc@126.com

College of Science, Civil Aviation University of China, Tianjin, 300300,

P.R. China

\begin{abstract}
We combine a sequence of contractive mappings $\left\{f_{n}\right\}$ and propose a generalized viscosity approximation method. One side, we consider a nonexpansive mapping $S$ with the nonempty fixed point set defined on a nonempty closed convex subset $C$ of a real Hilbert space $\mathrm{H}$ and design a new iterative method to approximate some fixed point of $S$, which is also a unique solution of the variational inequality. On the other hand, using similar ideas, we consider $N$ nonexpansive mappings $\left\{S_{i}\right\}_{i=1}^{N}$ with the nonempty common fixed point set defined on a nonempty closed convex subset $C$. Under reasonable conditions, strong convergence theorems are proven. The results presented in this paper improve and extend the corresponding results reported by some authors recently.

MSC: 47H09; 47H10;47J20; 47J25
\end{abstract}

Keywords: nonexpansive mapping; contractive mapping; variational inequality; fixed point; viscosity approximation method

\section{Introduction}

Let $H$ be a real Hilbert space with an inner product $\langle$,$\rangle and norm \|\cdot\|$, and $C$ be a nonempty closed convex subset of $H$.

Let $S: C \rightarrow C$ be a nonlinear mapping, we use $\operatorname{Fix}(S)$ to denote the set of fixed points of $S$ (i.e., $\operatorname{Fix}(S)=\{x \in C: S x=x\}$ ). A mapping is called nonexpansive if the following inequality holds:

$$
\|S x-S y\| \leq\|x-y\|
$$

for all $x, y \in C$.

In 1967, Halpern [1] used contractions to approximate a nonexpansive mapping and considered the following explicit iterative process:

$$
x_{0} \in C, \quad x_{n+1}=\alpha_{n} u+\left(1-\alpha_{n}\right) S x_{n}, \quad \forall n \geq 0,
$$

where $u$ is a given point and $S: C \rightarrow C$ is nonexpansive. He proved the strong convergence of $\left\{x_{n}\right\}$ to a fixed point of $S$ provided that $\alpha_{n}=n^{-\theta}$ with $\theta \in(0,1)$. In 2000, Moudafi [2] introduced the viscosity approximation method for nonexpansive mappings. Until now, in many references, viscosity approximation methods still are used and studied, which

\section{Springer}

(02014 Duan and He; licensee Springer. This is an Open Access article distributed under the terms of the Creative Commons Attribution License (http://creativecommons.org/licenses/by/2.0), which permits unrestricted use, distribution, and reproduction in any medium, provided the original work is properly cited. 
formally generates the sequence $\left\{x_{n}\right\}$ by the recursive formula:

$$
x_{n+1}=\alpha_{n} f\left(x_{n}\right)+\left(1-\alpha_{n}\right) S x_{n},
$$

where $f$ is a contraction and $\alpha_{n} \subset(0,1)$ is a slowly vanishing sequence. See, for instance, [3-6]. In fact, Yamada's hybrid steepest descent algorithm is also a kind of viscosity approximation method (see [7]).

The variational inequality problem is to find a point $x^{*} \in C$ such that

$$
\left\langle F x^{*}, x-x^{*}\right\rangle \geq 0, \quad \forall x \in C
$$

In recent years, the theory of variational inequality has been extended to the study of a large variety of problems arising in structural analysis, economics, engineering sciences, and so on. See [8-10] and the references cited therein.

Recently, Zhou and Wang [11] proposed a simpler explicit iterative algorithm for finding a solution of variational inequality over the set of common fixed points of a finite family nonexpansive mappings. They introduced an explicit scheme as follows.

Theorem 1.1 Let $H$ be a real Hilbert space and $F: H \rightarrow H$ be an L-Lipschitz continuous and $\eta$-strongly monotone mapping. Let $\left\{S_{i}\right\}_{i=1}^{N}$ be $N$ nonexpansive self-mappings of $H$ such that $C=\bigcap_{i=1}^{N} \operatorname{Fix}\left(S_{i}\right) \neq \emptyset$. For any point $x_{0} \in H$, define a sequence $\left\{x_{n}\right\}$ in the following manner:

$$
x_{n+1}=\left(I-\lambda_{n} \mu F\right) S_{N}^{n} S_{N-1}^{n} \cdots S_{1}^{n} x_{n}, \quad n \geq 0,
$$

where $\mu \in\left(0,2 \eta / L^{2}\right)$ and $S_{i}^{n}:=\left(1-\beta_{n}^{i}\right) I+\beta_{n}^{i} S_{i}$ for $i=1,2, \ldots, N$. When the parameters satisfy appropriate conditions, the sequence converges strongly to the unique solution of the variational inequality (1.1).

In this paper, motivated by the above works, we introduce a more generalized iterative method like viscosity approximation. In Section 3, we combine a sequence of contractive mappings and obtain strong convergence theorem for approximating fixed point of a nonexpansive mapping. In Section 4, we propose a new iterative algorithm for finding some common fixed point of a finite family nonexpansive mappings, which is also a unique solution for the variational inequality over the set of fixed point of these mappings on Hilbert spaces.

\section{Preliminaries}

In order to prove our results, we collect some facts and tools in a real Hilbert space $H$, which are listed as below.

Lemma 2.1 Let $H$ be a real Hilbert space. We have the following inequalities:

(i) $\|x+y\|^{2} \leq\|x\|^{2}+2\langle x+y, y\rangle, \forall x, y \in H$.

(ii) $\|t x+(1-t) y\|^{2} \leq t\|x\|^{2}+(1-t)\|y\|^{2}, \forall t \in[0,1], \forall x, y \in H$.

Lemma 2.2 [12] Let $\left\{S_{i}\right\}_{i=1}^{2}$ be $\gamma_{i}$-averaged on $C$ such that $\operatorname{Fix}\left(S_{1}\right) \cap \operatorname{Fix}\left(S_{2}\right) \neq \emptyset$. Then the following conclusions hold: 
(i) both $S_{1} S_{2}$ and $S_{2} S_{1}$ are $\gamma$-averaged, where $\gamma=\gamma_{1}+\gamma_{2}-\gamma_{1} \gamma_{2}$;

(ii) $\operatorname{Fix}\left(S_{1}\right) \cap \operatorname{Fix}\left(S_{2}\right)=\operatorname{Fix}\left(S_{1} S_{2}\right)=\operatorname{Fix}\left(S_{2} S_{1}\right)$.

Recall that given a nonempty closed convex subset $C$ of a real Hilbert space $H$, for any $x \in H$, there exists a unique nearest point in $C$, denoted by $P_{C} x$, such that

$$
\left\|x-P_{C} x\right\| \leq\|x-y\|
$$

for all $y \in C$. Such a $P_{C}$ is called the metric (or the nearest point) projection of $H$ onto $C$.

Lemma 2.3 [13] Let $C$ be a nonempty closed convex subset of a real Hilbert space $H$. Given $x \in H$ and $z \in C$, then $y=P_{C} x$ if and only if we have the relation

$$
\langle x-y, y-z\rangle \geq 0 \quad \text { for all } z \in C \text {. }
$$

Lemma 2.4 [10] Let $H$ be a Hilbert space and $C$ be a nonempty closed convex subset of $H$, and $T: C \rightarrow C$ a nonexpansive mapping with $\operatorname{Fix}(T) \neq \emptyset$. If $\left\{x_{n}\right\}$ is a sequence in $C$ weakly converging to $x$ and if $\left\{(I-T) x_{n}\right\}$ converges strongly to $y$, then $(I-T) x=y$.

Lemma 2.5 [5] Assume that $\left\{a_{n}\right\}$ is a sequence of nonnegative real numbers such that

$$
a_{n+1} \leq\left(1-\gamma_{n}\right) a_{n}+\delta_{n}
$$

where $\left\{\gamma_{n}\right\}$ is a sequence in $(0,1)$ and $\left\{\delta_{n}\right\}$ is a sequence such that

$$
\begin{aligned}
& \text { (i) } \sum_{n=1}^{\infty} \gamma_{n}=\infty ; \\
& \text { (ii) } \quad \limsup _{n \rightarrow \infty} \frac{\delta_{n}}{\gamma_{n}} \leq 0 \text { or } \sum_{n=1}^{\infty}\left|\delta_{n}\right|<\infty .
\end{aligned}
$$

Then, $\lim _{n \rightarrow \infty} a_{n}=0$.

Lemma 2.6 [14] Let $\left\{x_{n}\right\}$ and $\left\{z_{n}\right\}$ be bounded sequences in a Banach space and $\left\{\beta_{n}\right\}$ be a sequence of real numbers such that $0<\liminf _{n \rightarrow \infty} \beta_{n} \leq \limsup _{n \rightarrow \infty} \beta_{n}<1$ for all $n=$ $0,1,2, \ldots$ Suppose that $x_{n+1}=\left(1-\beta_{n}\right) z_{n}+\beta_{n} x_{n}$ for all $n=0,1,2, \ldots$ and $\lim \sup _{n \rightarrow \infty}\left(\| z_{n+1}-\right.$ $\left.z_{n}\|-\| x_{n+1}-x_{n} \|\right) \leq 0$. Then $\lim _{n \rightarrow \infty}\left\|z_{n}-x_{n}\right\|=0$.

\section{Generalized viscosity approximation method combining with a nonexpansive mapping}

In this section, we combine a sequence of contractive mappings and apply a more generalized iterative method like viscosity approximation to approximate some fixed point of a nonexpansive mapping defined on a closed convex subset $C$ of a Hilbert space $H$, which is also the solution of the variational inequality

$$
\left\langle f\left(x^{*}\right)-x^{*}, p-x^{*}\right\rangle \leq 0, \quad \forall p \in \operatorname{Fix}(S) .
$$


Suppose the contractive mapping sequence $\left\{f_{n}(x)\right\}$ is uniformly convergent for any $x \in D$, where $D$ is any bounded subset of $C$. The uniform convergence of $\left\{f_{n}(x)\right\}$ on $D$ is denoted by $f_{n}(x) \rightrightarrows f(x)(n \rightarrow \infty), x \in D$.

Theorem 3.1 Let $C$ be a nonempty closed convex subset of a real Hilbert space $H$ and let $\left\{f_{n}\right\}$ be a sequence of $\rho_{n}$-contractive self-maps of $C$ with $0 \leq \rho_{l}=\liminf _{n \rightarrow \infty} \rho_{n} \leq$ $\lim \sup _{n \rightarrow \infty} \rho_{n}=\rho_{u}<1$. Let $S: C \rightarrow C$ be a nonexpansive mapping. Assume the set $\operatorname{Fix}(S) \neq$ $\emptyset$ and $\left\{f_{n}(x)\right\}$ is uniformly convergent for any $x \in D$, where $D$ is any bounded subset of $C$. Given $x_{1} \in C$, let $\left\{x_{n}\right\}$ be generated by the following algorithm:

$$
x_{n+1}=\alpha_{n} f_{n}\left(x_{n}\right)+\left(1-\alpha_{n}\right) S x_{n} .
$$

If the sequence $\left\{\alpha_{n}\right\} \subset(0,1)$ satisfies the following conditions:

(i) $\lim _{n \rightarrow \infty} \alpha_{n}=0$;

(ii) $\sum_{n=1}^{\infty} \alpha_{n}=\infty$;

(iii) $\sum_{n=1}^{\infty}\left|\alpha_{n+1}-\alpha_{n}\right|<\infty$,

then the sequence $\left\{x_{n}\right\}$ converges strongly to a point $x^{*} \in \operatorname{Fix}(S)$, which is also the unique solution of the variational inequality (3.1).

Proof The proof is divided into several steps.

Step 1. Show first that $\left\{x_{n}\right\}$ is bounded.

For any $q \in \operatorname{Fix}(S)$, we have

$$
\begin{aligned}
\left\|x_{n+1}-q\right\| & =\left\|\alpha_{n} f_{n}\left(x_{n}\right)+\left(1-\alpha_{n}\right) S x_{n}-q\right\| \\
& \leq \alpha_{n}\left\|f_{n}\left(x_{n}\right)-q\right\|+\left(1-\alpha_{n}\right)\left\|S x_{n}-S q\right\| \\
& \leq \alpha_{n} \rho_{n}\left\|x_{n}-q\right\|+\left(1-\alpha_{n}\right)\left\|x_{n}-q\right\|+\alpha_{n}\left\|f_{n}(q)-q\right\| \\
& \leq\left(1-\alpha_{n}\left(1-\rho_{n}\right)\right)\left\|x_{n}-q\right\|+\alpha_{n}\left(1-\rho_{n}\right) \frac{\left\|f_{n}(q)-q\right\|}{1-\rho_{n}} \\
& \leq \max \left\{\left\|x_{n}-q\right\|, \frac{\left\|f_{n}(q)-q\right\|}{1-\rho_{n}}\right\} .
\end{aligned}
$$

From the uniform convergence of $\left\{f_{n}\right\}$ on $D$, it is easy to get the boundedness of $\left\{f_{n}(q)\right\}$. Thus there exists a positive constant $M_{1}$, such that $\left\|f_{n}(q)-q\right\| \leq M_{1}$. By induction, we obtain $\left\|x_{n}-p\right\| \leq \max \left\{\left\|x_{1}-p\right\|, \frac{M_{1}}{1-\rho_{u}}\right\}$. Hence, $\left\{x_{n}\right\}$ is bounded, so are $\left\{S x_{n}\right\}$ and $\left\{f_{n}\left(x_{n}\right)\right\}$.

Step 2. Show that

$$
\left\|x_{n+1}-x_{n}\right\| \rightarrow 0 \quad \text { as } n \rightarrow \infty \text {. }
$$

Indeed, observe that

$$
\begin{aligned}
\left\|x_{n+1}-x_{n}\right\|= & \left\|\alpha_{n} f_{n}\left(x_{n}\right)+\left(1-\alpha_{n}\right) S x_{n}-\alpha_{n-1} f_{n-1}\left(x_{n-1}\right)-\left(1-\alpha_{n-1}\right) S x_{n-1}\right\| \\
= & \| \alpha_{n}\left(f_{n}\left(x_{n}\right)-f_{n}\left(x_{n-1}\right)\right)+\alpha_{n}\left(f_{n}\left(x_{n-1}\right)-f_{n-1}\left(x_{n-1}\right)\right) \\
& +\left(\alpha_{n}-\alpha_{n-1}\right)\left(f_{n-1}\left(x_{n-1}\right)-S x_{n-1}\right)+\left(1-\alpha_{n}\right)\left(S x_{n}-S x_{n-1}\right) \| \\
\leq & \alpha_{n} \rho_{n}\left\|x_{n}-x_{n-1}\right\|+\alpha_{n}\left\|f_{n}\left(x_{n-1}\right)-f_{n-1}\left(x_{n-1}\right)\right\|
\end{aligned}
$$




$$
\begin{aligned}
& +\left|\alpha_{n}-\alpha_{n-1}\right|\left(\left\|S x_{n}\right\|+\left\|f_{n-1}\left(x_{n-1}\right)\right\|\right)+\left(1-\alpha_{n}\right)\left\|x_{n}-x_{n-1}\right\| \\
= & \left(1-\alpha_{n}\left(1-\rho_{n}\right)\right)\left\|x_{n}-x_{n-1}\right\|+\alpha_{n}\left\|f_{n}\left(x_{n-1}\right)-f_{n-1}\left(x_{n-1}\right)\right\| \\
& +\left|\alpha_{n}-\alpha_{n-1}\right|\left(\left\|S x_{n}\right\|+\left\|f_{n-1}\left(x_{n-1}\right)\right\|\right) .
\end{aligned}
$$

By the conditions (i)-(iii) and the uniform convergence of $f_{n}(x)$, we have

$$
\frac{\alpha_{n}\left\|f_{n}\left(x_{n-1}\right)-f_{n-1} x_{n-1}\right\|+\left|\alpha_{n}-\alpha_{n-1}\right|\left(\left\|S x_{n}\right\|+\left\|f_{n-1} x_{n-1}\right\|\right)}{\alpha_{n}\left(1-\rho_{n}\right)} \rightarrow 0
$$

as $n \rightarrow \infty$. By Lemma 2.5, (3.3) holds.

Step 3. Show that

$$
\left\|S x_{n}-x_{n}\right\| \rightarrow 0
$$

Since

$$
\left\|S x_{n}-x_{n}\right\| \leq\left\|x_{n+1}-x_{n}\right\|+\left\|x_{n+1}-S x_{n}\right\| .
$$

By the condition (i), we have $\left\|x_{n+1}-S x_{n}\right\|=\alpha_{n}\left\|f_{n}\left(x_{n}\right)-S x_{n}\right\| \rightarrow 0$. Combining with (3.3), it is easy to get (3.4).

Step 4.

$$
\limsup _{n \rightarrow \infty}\left\langle f\left(x^{*}\right)-x^{*}, x_{n}-x^{*}\right\rangle \leq 0
$$

where $x^{*}=P_{\operatorname{Fix}(S)} f\left(x^{*}\right)$ is a unique solution of the variational inequality (3.1).

Since $f_{n}(x)$ is uniformly convergent on $D$, we have $\lim _{n \rightarrow \infty}\left(f_{n}\left(x^{*}\right)-x^{*}\right)=f\left(x^{*}\right)-x^{*}$.

Indeed, take a subsequence $\left\{x_{n_{j}}\right\}$ of $\left\{x_{n}\right\}$ such that

$$
\limsup _{n \rightarrow \infty}\left\langle f\left(x^{*}\right)-x^{*}, x_{n}-x^{*}\right\rangle=\lim _{j \rightarrow \infty}\left\langle f\left(x^{*}\right)-x^{*}, x_{n_{j}}-x^{*}\right\rangle .
$$

Since $\left\{x_{n_{j}}\right\}$ is bounded, there exists a subsequence $\left\{x_{n_{j_{k}}}\right\}$ of $\left\{x_{n_{j}}\right\}$ which converges weakly to $\hat{x}$. Without loss of generality, we can assume $x_{n_{j}} \rightarrow \hat{x}$. From (3.4), we obtain $S x_{n_{j}}-\hat{x}$. Using Lemma 2.4, we have $\hat{x} \in \operatorname{Fix}(S)$. Since $x^{*}=P_{\operatorname{Fix}(S)} f\left(x^{*}\right)$, we get

$$
\lim _{j \rightarrow \infty}\left\langle f\left(x^{*}\right)-x^{*}, x_{n_{j}}-x^{*}\right\rangle=\left\langle f\left(x^{*}\right)-x^{*}, \hat{x}-x^{*}\right\rangle \leq 0
$$

Combining with (3.6), the inequality (3.5) holds.

Step 5. Show that

$$
\begin{aligned}
& x_{n} \rightarrow x^{*}, \\
& \begin{aligned}
\left\|x_{n+1}-x^{*}\right\|^{2} \\
\quad=\left\|\alpha_{n} f_{n}\left(x_{n}\right)+\left(1-\alpha_{n}\right) S x_{n}-x^{*}\right\|^{2} \\
\quad \leq\left(1-\alpha_{n}\right)^{2}\left\|S x_{n}-x^{*}\right\|^{2}+2 \alpha_{n}\left(x_{n+1}-x^{*}, f_{n}\left(x_{n}\right)-x^{*}\right\rangle
\end{aligned}
\end{aligned}
$$




$$
\begin{aligned}
\leq & \left(1-\alpha_{n}\right)^{2}\left\|x_{n}-x^{*}\right\|^{2}+2 \alpha_{n}\left\langle x_{n+1}-x^{*}, f_{n}\left(x_{n}\right)-f_{n}\left(x^{*}\right)\right\rangle+2 \alpha_{n}\left\langle x_{n+1}-x^{*}, f_{n}\left(x^{*}\right)-x^{*}\right\rangle \\
\leq & \left(1-\alpha_{n}\right)^{2}\left\|x_{n}-x^{*}\right\|^{2}+\alpha_{n} \rho_{n}\left(\left\|x_{n}-x^{*}\right\|^{2}+\left\|x_{n+1}-x^{*}\right\|^{2}\right) \\
& +2 \alpha_{n}\left\langle x_{n+1}-x^{*}, f_{n}\left(x^{*}\right)-x^{*}\right\rangle .
\end{aligned}
$$

Transform the inequality into another form, we obtain

$$
\left\|x_{n+1}-x^{*}\right\|^{2} \leq\left(1-\frac{\alpha_{n}\left(2-\alpha_{n}-2 \rho_{n}\right)}{1-\alpha_{n} \rho_{n}}\right)\left\|x_{n}-x^{*}\right\|^{2}+\frac{2 \alpha_{n}}{1-\alpha_{n} \rho_{n}}\left\langle x_{n+1}-x^{*}, f_{n}\left(x^{*}\right)-x^{*}\right\rangle .
$$

By Schwartz's inequality, we have

$$
\begin{aligned}
& \limsup _{n \rightarrow \infty}\left\langle x_{n+1}-x^{*}, f_{n}\left(x^{*}\right)-x^{*}\right\rangle \\
& \quad \leq \lim _{n \rightarrow \infty}\left\|x_{n+1}-x^{*}\right\|\left\|f_{n}\left(x^{*}\right)-f\left(x^{*}\right)\right\|+\limsup _{n \rightarrow \infty}\left\langle x_{n+1}-x^{*}, f\left(x^{*}\right)-x^{*}\right\rangle .
\end{aligned}
$$

By the boundedness of $\left\{x_{n}\right\}, f_{n}(x) \rightrightarrows f(x),(3.3)$ and (3.5), we have

$$
\limsup _{n \rightarrow \infty}\left\langle x_{n+1}-x^{*}, f_{n}\left(x^{*}\right)-x^{*}\right\rangle \leq 0
$$

It follows from Lemma 2.5 that (3.7) holds.

Remark 3.2 In [2], Moudafi proposed the viscosity iterative algorithm as follows:

$$
x_{n+1}=\alpha_{n} f\left(x_{n}\right)+\left(1-\alpha_{n}\right) S x_{n},
$$

where $f$ is a contraction on $H$. It is a special case of (3.2) in this paper when $f_{1}=f_{2}=\cdots=$ $f_{n}=\cdots=f, \forall n \in \mathbb{N}$ and $C=H$. Of course, Halpern's iteration method is also a special case of (3.2) when $f_{1}=f_{2}=\cdots=f_{n}=\cdots=u, \forall n \in \mathbb{N}$.

Remark 3.3 In [7], the following iterative process was introduced:

$$
x_{n+1}=S x_{n}-\mu \alpha_{n} F\left(S x_{n}\right) .
$$

Rewriting the equation, we get

$$
\begin{aligned}
x_{n+1} & =\alpha_{n}(I-\mu F) S x_{n}+\left(1-\alpha_{n}\right) S x_{n} \\
& =\alpha_{n} f\left(x_{n}\right)+\left(1-\alpha_{n}\right) S x_{n} .
\end{aligned}
$$

It is easily to verify $f:=(I-\mu F) S$ is a contractive mapping on $H$ when $0<\mu<2 \eta / L^{2}$. That is, Yamada's method is a kind of viscosity approximation method. Of course it is also a special case of Theorem 3.1.

\section{Generalized viscosity approximation method combining with a finite family of nonexpansive mappings}

In this section, we apply a more generalized iterative method like viscosity approximation to approximate a common element of the set of fixed points of a finite family of nonexpansive mappings on Hilbert spaces. 
Let $\left\{f_{n}\right\}$ be a sequence of $\rho_{n}$-contractive self-maps of $C$ with $0<\rho_{l}=\liminf _{n \rightarrow \infty} \rho_{n} \leq$ $\limsup _{n \rightarrow \infty} \rho_{n}=\rho_{u}<1$ and $\left\{S_{i}\right\}_{i=1}^{N}$ be $N$ nonexpansive self-mapping of $C$. Assume the common fixed point set $F=\bigcap_{i=1}^{N} \operatorname{Fix}\left(S_{i}\right) \neq \emptyset$ and $\left\{f_{n}(q)\right\}$ is convergent for any $q \in F$. Put $f(q):=\lim _{n \rightarrow \infty} f_{n}(q)$, since every $f_{n}$ is $\rho_{n}$-contractive, we have

$$
\left\|f_{n}(p)-f_{n}(q)\right\| \leq \rho_{n}\|p-q\| \leq \rho_{u}\|p-q\|
$$

for any $p, q \in F$. Further we obtain $\|f(p)-f(q)\| \leq \rho_{u}\|p-q\|$. Next we prove the sequence $\left\{x_{n}\right\}$ converges strongly to a point $x^{*} \in F=\bigcap_{i=1}^{N} \operatorname{Fix}\left(S_{i}\right)$, which also solves the variational inequality

$$
\left\langle f\left(x^{*}\right)-x^{*}, p-x^{*}\right\rangle \leq 0, \quad \forall p \in F .
$$

As we know, it is equivalent to the fixed point equation $x^{*}=P_{F} f\left(x^{*}\right)$.

Theorem 4.1 Let $C$ be a nonempty closed convex subset of a real Hilbert space $H$ and let $\left\{f_{n}\right\}$ be a sequence of $\rho_{n}$-contractive self-maps of $C$ with $0 \leq \rho_{l}=\liminf _{n \rightarrow \infty} \rho_{n} \leq$ $\limsup _{n \rightarrow \infty} \rho_{n}=\rho_{u}<1$. Let, for each $1 \leq i \leq N$ ( $N \geq 1$ be an integer), $S_{i}: C \rightarrow C$ be a nonexpansive mapping. Assume the set $F=\bigcap_{i=1}^{N} \operatorname{Fix}\left(S_{i}\right) \neq \emptyset$ and $\left\{f_{n}(q)\right\}$ is convergent for any $q \in F$. Given $x_{1} \in C$, let $\left\{x_{n}\right\}$ be generated by the following algorithm:

$$
\left\{\begin{array}{l}
x_{n+1}=\alpha_{n} f_{n}\left(x_{n}\right)+\left(1-\alpha_{n}\right) S_{N}^{n} S_{N-1}^{n} \cdots S_{1}^{n} x_{n}, \\
S_{i}^{n}=\left(1-\lambda_{i}^{n}\right) I+\lambda_{i}^{n} S_{i}, \quad i=1,2, \ldots, N .
\end{array}\right.
$$

If the parameters $\left\{\alpha_{n}\right\}$ and $\left\{\lambda_{i}^{n}\right\}$ satisfy the following conditions:

(i) $\left\{\alpha_{n}\right\} \subset(0,1), \lim _{n \rightarrow \infty} \alpha_{n}=0$ and $\sum_{n=1}^{\infty} \alpha_{n}=\infty$;

(ii) $\lambda_{i}^{n} \in\left(\lambda_{l}, \lambda_{u}\right)$ for some $\lambda_{l}, \lambda_{u} \in(0,1)$ and $\lim _{n \rightarrow \infty}\left|\lambda_{i}^{n}-\lambda_{i}^{n+1}\right|=0, \forall i=1,2, \ldots, N$, then the sequence $\left\{x_{n}\right\}$ converges strongly to a point $x^{*} \in F$, which is also the unique solution of the variational inequality (4.1).

Proof We will prove the theorem in the case of $N=2$. The proof is divided into several steps.

Step 1. We show first that $\left\{x_{n}\right\}$ is bounded.

For any $q \in F$, we have

$$
\begin{aligned}
\left\|x_{n+1}-q\right\| & =\left\|\alpha_{n} f_{n}\left(x_{n}\right)+\left(1-\alpha_{n}\right) S_{2}^{n} S_{1}^{n} x_{n}-q\right\| \\
& \leq \alpha_{n}\left\|f_{n}\left(x_{n}\right)-q\right\|+\left(1-\alpha_{n}\right)\left\|S_{2}^{n} S_{1}^{n} x_{n}-S_{2}^{n} S_{1}^{n} q\right\| \\
& \leq \alpha_{n} \rho_{n}\left\|x_{n}-q\right\|+\left(1-\alpha_{n}\right)\left\|x_{n}-q\right\|+\alpha_{n}\left\|f_{n}(q)-q\right\| \\
& \leq\left(1-\alpha_{n}\left(1-\rho_{n}\right)\right)\left\|x_{n}-q\right\|+\alpha_{n}\left(1-\rho_{n}\right) \frac{\left\|f_{n}(q)-q\right\|}{1-\rho_{n}} \\
& \leq \max \left\{\left\|x_{n}-q\right\|, \frac{\left\|f_{n}(q)-q\right\|}{1-\rho_{n}}\right\} .
\end{aligned}
$$

From the convergence of $\left\{f_{n}(q)\right\}$, it is easy to get the boundness of $\left\{f_{n}(q)\right\}$. Thus there exists a positive constant $M_{1}$, such that $\left\|f_{n}(q)-q\right\| \leq M_{1}$. By induction, we obtain $\left\|x_{n}-p\right\| \leq$ $\max \left\{\left\|x_{1}-p\right\|, \frac{M_{1}}{1-\rho_{u}}\right\}$. Hence, $\left\{x_{n}\right\}$ is bounded, and so are $\left\{S_{1} x_{n}\right\}$ and $\left\{S_{2}^{n} S_{1}^{n} x_{n}\right\}$. 
Step 2. We show that

$$
\left\|x_{n+1}-x_{n}\right\| \rightarrow 0 \quad \text { as } n \rightarrow \infty
$$

Since both $S_{2}^{n}$ and $S_{1}^{n}$ are averaged nonexpansive mappings, by Lemma 2.2, $S_{2}^{n} S_{1}^{n}$ is also averaged. Rewrite $S_{2}^{n} S_{1}^{n}=\left(1-\beta_{n}\right) I+\beta_{n} V_{n}$, where $\beta_{n}=\lambda_{1}^{n}+\lambda_{2}^{n}-\lambda_{1}^{n} \lambda_{2}^{n}$. Then we have

$$
\begin{aligned}
x_{n+1} & =\alpha_{n} f_{n}\left(x_{n}\right)+\left(1-\alpha_{n}\right)\left[\left(1-\beta_{n}\right) I+\beta_{n} V_{n}\right] x_{n} \\
& =\alpha_{n} f_{n}\left(x_{n}\right)+\left(1-\beta_{n}\right) x_{n}-\alpha_{n}\left(1-\beta_{n}\right) x_{n}+\left(1-\alpha_{n}\right) \beta_{n} V_{n} x_{n} \\
& =\left(1-\beta_{n}\right) x_{n}+\beta_{n}\left[\alpha_{n} \frac{f_{n}\left(x_{n}\right)-\left(1-\beta_{n}\right) x_{n}}{\beta_{n}}+\left(1-\alpha_{n}\right) V_{n} x_{n}\right] \\
& =\left(1-\beta_{n}\right) x_{n}+\beta_{n} z_{n} .
\end{aligned}
$$

Further we obtain

$$
\begin{aligned}
\| z_{n+1} & -z_{n} \| \\
= & \| \frac{\alpha_{n+1}}{\beta_{n+1}}\left[f_{n+1}\left(x_{n+1}\right)-\left(1-\beta_{n+1}\right) x_{n+1}\right]+\left(1-\alpha_{n+1}\right) V_{n+1} x_{n+1} \\
& -\frac{\alpha_{n}}{\beta_{n}}\left[f_{n}\left(x_{n}\right)-\left(1-\beta_{n}\right) x_{n}\right]-\left(1-\alpha_{n}\right) V_{n} x_{n} \| \\
= & \left\|V_{n+1} x_{n+1}-V_{n} x_{n}\right\|+\|\left[\frac{\alpha_{n+1}}{\beta_{n+1}} f_{n+1}\left(x_{n+1}\right)-\frac{\alpha_{n}}{\beta_{n}} f_{n}\left(x_{n}\right)\right] \\
& -\left[\frac{\alpha_{n+1}\left(1-\beta_{n+1}\right)}{\beta_{n+1}} x_{n+1}-\frac{\alpha_{n}\left(1-\beta_{n}\right)}{\beta_{n}} x_{n}\right]-\alpha_{n+1} V_{n+1} x_{n+1}+\alpha_{n} V_{n} x_{n} \| \\
\leq & \left\|x_{n+1}-x_{n}\right\|+\left\|V_{n+1} x_{n}-V_{n} x_{n}\right\|+\left|\frac{\alpha_{n+1}}{\beta_{n+1}} f_{n+1}\left(x_{n+1}\right)-\frac{\alpha_{n}}{\beta_{n}} f_{n}\left(x_{n}\right)\right| \\
& +\left\|\frac{\alpha_{n+1}\left(1-\beta_{n+1}\right)}{\beta_{n+1}} x_{n+1}-\frac{\alpha_{n}\left(1-\beta_{n}\right)}{\beta_{n}} x_{n}\right\| \\
& +\left\|\alpha_{n+1} V_{n+1} x_{n+1}-\alpha_{n} V_{n} x_{n}\right\| .
\end{aligned}
$$

Write $\lambda_{1}=2 \lambda_{l}-\lambda_{l}^{2}, \lambda_{2}=2 \lambda_{u}-\lambda_{u}^{2}$. From the condition (iii), it is easily to get $0<\lambda_{1} \leq \beta_{n} \leq \lambda_{2}$ and $\beta_{n+1}-\beta_{n} \rightarrow 0$ as $n \rightarrow \infty$. We have

$$
\begin{aligned}
\left\|V_{n+1} x_{n}-V_{n} x_{n}\right\|= & \left\|\frac{S_{2}^{n+1} S_{1}^{n+1}-\left(1-\beta_{n+1}\right) I}{\beta_{n+1}} x_{n}-\frac{S_{2}^{n} S_{1}^{n}-\left(1-\beta_{n}\right) I}{\beta_{n}} x_{n}\right\| \\
\leq & \left\|\frac{S_{2}^{n+1} S_{1}^{n+1}}{\beta_{n+1}} x_{n}-\frac{S_{2}^{n} S_{1}^{n}}{\beta_{n}} x_{n}\right\|+\left|\frac{1}{\beta_{n}}-\frac{1}{\beta_{n+1}}\right|\left\|x_{n}\right\| \\
\leq & \frac{1}{\beta_{n}}\left\|S_{2}^{n+1} S_{1}^{n+1} x_{n}-S_{2}^{n} S_{1}^{n} x_{n}\right\|+\left|\frac{1}{\beta_{n}}-\frac{1}{\beta_{n+1}}\right|\left(\left\|S_{2}^{n+1} S_{1}^{n+1} x_{n}\right\|+\left\|x_{n}\right\|\right) \\
\leq & \frac{1}{\lambda_{1}}\left(\left\|S_{1}^{n+1} x_{n}-S_{1}^{n} x_{n}\right\|+\left\|S_{2}^{n+1} S_{1}^{n} x_{n}-S_{2}^{n} S_{1}^{n} x_{n}\right\|\right) \\
& +\left|\frac{1}{\beta_{n}}-\frac{1}{\beta_{n+1}}\right|\left(\left\|S_{2}^{n+1} S_{1}^{n+1} x_{n}\right\|+\left\|x_{n}\right\|\right)
\end{aligned}
$$




$$
\begin{aligned}
\leq & \frac{1}{\lambda_{1}}\left(\left\|S_{1}^{n+1} x_{n}-S_{1}^{n} x_{n}\right\|+\left\|S_{2}^{n+1} S_{1}^{n} x_{n}-S_{2}^{n} S_{1}^{n} x_{n}\right\|\right) \\
& +\left|\frac{1}{\beta_{n}}-\frac{1}{\beta_{n+1}}\right| M_{2}
\end{aligned}
$$

where $M_{2}=\sup _{n}\left\{\left\|S_{2}^{n+1} S_{1}^{n+1} x_{n}\right\|+\left\|x_{n}\right\|\right\}$. Since $\left|\lambda_{i}^{n+1}-\lambda_{i}^{n}\right| \rightarrow 0, i=1,2$, we can deduce

$$
\left\|S_{1}^{n+1} x_{n}-S_{1}^{n} x_{n}\right\| \leq\left|\lambda_{1}^{n+1}-\lambda_{1}^{n}\right|\left(\left\|x_{n}\right\|+\left\|S_{1} x_{n}\right\|\right) \rightarrow 0
$$

and

$$
\left\|S_{2}^{n+1} S_{1}^{n} x_{n}-S_{2}^{n} S_{1}^{n} x_{n}\right\| \leq\left|\lambda_{2}^{n+1}-\lambda_{2}^{n}\right|\left(\left\|S_{1}^{n} x_{n}\right\|+\left\|S_{2} S_{1}^{n} x_{n}\right\|\right) \rightarrow 0
$$

Substituting (4.5) into (4.4), we have

$$
\begin{aligned}
& \left\|z_{n+1}-z_{n}\right\|-\left\|x_{n+1}-x_{n}\right\| \\
& \leq \frac{1}{\lambda_{1}}\left(\left\|S_{1}^{n+1} x_{n}-S_{1}^{n} x_{n}\right\|+\left\|S_{2}^{n+1} S_{1}^{n} x_{n}-S_{2}^{n} S_{1}^{n} x_{n}\right\|\right)+\frac{\left|\beta_{n}-\beta_{n+1}\right|}{\beta_{n} \beta_{n+1}} M_{2} \\
& \quad+\left\|\frac{\alpha_{n+1}}{\beta_{n+1}} f_{n+1}\left(x_{n+1}\right)-\frac{\alpha_{n}}{\beta_{n}} f_{n}\left(x_{n}\right)\right\|+\left\|\frac{\alpha_{n+1}\left(1-\beta_{n+1}\right)}{\beta_{n+1}} x_{n+1}-\frac{\alpha_{n}\left(1-\beta_{n}\right)}{\beta_{n}} x_{n}\right\| \\
& \quad+\left\|\alpha_{n+1} V_{n+1} x_{n+1}-\alpha_{n} V_{n} x_{n}\right\| .
\end{aligned}
$$

Combining (4.6), (4.7), and condition (i), we get

$$
\limsup _{n \rightarrow \infty}\left(\left\|z_{n+1}-z_{n}\right\|-\left\|x_{n+1}-x_{n}\right\|\right) \leq 0
$$

By Lemma 2.6, we conclude that $\lim _{n \rightarrow \infty}\left\|z_{n}-x_{n}\right\| \rightarrow 0$. Further we have

$$
\lim _{n \rightarrow \infty}\left\|x_{n+1}-x_{n}\right\|=\lim _{n \rightarrow \infty} \beta_{n}\left\|z_{n}-x_{n}\right\| \rightarrow 0 .
$$

Step 3. We show that

$$
\left\|S_{2}^{n} S_{1}^{n} x_{n}-x_{n}\right\| \rightarrow 0
$$

By (4.2), we get

$$
\left\|x_{n+1}-S_{2}^{n} S_{1}^{n} x_{n}\right\|=\alpha_{n}\left\|f_{n}\left(x_{n}\right)-S_{2}^{n} S_{1}^{n} x_{n}\right\| \rightarrow 0
$$

We have

$$
\left\|x_{n}-S_{2}^{n} S_{1}^{n} x_{n}\right\| \leq\left\|x_{n+1}-S_{2}^{n} S_{1}^{n} x_{n}\right\|+\left\|x_{n}-x_{n+1}\right\| .
$$

Combining with (4.3), (4.8) holds.

Since $\left\{\lambda_{i}^{n}\right\} \subset\left(\lambda_{l}, \lambda_{u}\right)$, we can assume that $\lambda_{i}^{n_{j}} \rightarrow \lambda_{i}^{0}$ as $n \rightarrow \infty$. It is easy to get $0<\lambda_{i}^{0}<1$ for $i=1,2$. Write $S_{i}^{0}=\left(1-\lambda_{i}^{0}\right) I+\lambda_{i}^{0} S_{i}, i=1,2$. Then we have $\operatorname{Fix}\left(S_{i}^{0}\right)=\operatorname{Fix}\left(S_{i}\right), i=1,2$ and

$$
\lim _{j \rightarrow \infty} \sup _{x \in D}\left\|S_{i}^{n_{j}} x-S_{i}^{0} x\right\|=0
$$


where $D$ is an arbitrary bounded subset including $\left\{x_{n_{j}}\right\}$. By using (4.8) and (4.9), we obtain $\left\|S_{2}^{0} S_{1}^{0} x_{n}-x_{n}\right\| \rightarrow 0$.

Step 4. We have

$$
\limsup _{n \rightarrow \infty}\left\{f\left(x^{*}\right)-x^{*}, x_{n}-x^{*}\right\rangle \leq 0
$$

where $x^{*}=P_{F} f\left(x^{*}\right)$ is a unique solution of the variational inequality (4.1).

Since $f_{n}(q)$ is convergent, we have $\lim _{n \rightarrow \infty}\left(f_{n}\left(x^{*}\right)-x^{*}\right)=f\left(x^{*}\right)-x^{*}$.

The proof of Step 4 is similar to that of Theorem 3.1.

Step 5. We show that

$$
x_{n} \rightarrow x^{*} .
$$

The proof of Step 5 is similar to that of Theorem 3.1.

Remark 4.2 In [11], put $S_{n}=S_{N}^{n} S_{N-1}^{n} \cdots S_{1}^{n}$, and we rewrite Zhou and Wang's iterative algorithm as follows:

$$
\begin{aligned}
x_{n+1} & =\left(I-\alpha_{n} \mu F\right) S_{n} x_{n} \\
& =\alpha_{n}(I-\mu F) S_{n} x_{n}+\left(1-\alpha_{n}\right) S_{n} x_{n} \\
& =\alpha_{n} f_{n}\left(x_{n}\right)+\left(1-\alpha_{n}\right) S_{n} x_{n} .
\end{aligned}
$$

It is easily to verify $(I-\mu F) S_{n}$ is a contractive mapping on $H$ when $0<\mu<2 \eta / L^{2}$. Thus it is a special case of Theorem 4.1 when $f_{n}:=(I-\mu F) S_{n}, \forall n \in N$ and $C=H$.

\section{Competing interests}

The authors declare that they have no competing interests.

\section{Authors' contributions}

All authors contributed equally to the writing of this paper. All authors read and approved the final manuscript.

\section{Acknowledgements}

The authors would like to thank the referee for valuable suggestions to improve the manuscript and the Fundamental Research Funds for the Central Universities (GRANT: 3122013k004).

Received: 30 October 2013 Accepted: 6 March 2014 Published: 20 Mar 2014

\section{References}

1. Halpern, B: Fixed points of nonexpanding maps. Bull. Am. Math. Soc. 73, 957-961 (1967)

2. Moudafi, A: Viscosity approximation methods for fixed-points problems. J. Math. Anal. Appl. 241, 46-55 (2000)

3. Chen, J, Zhang, L, Fan, T: Viscosity approximation methods for nonexpansive mappings and monotone mappings. J. Math. Anal. Appl. 334, 1450-1461 (2007)

4. Takahashi, W: Viscosity approximation methods for countable families of nonexpansive mappings in Banach spaces. Nonlinear Anal. 70, 719-734 (2009)

5. Xu, HK: Viscosity approximation methods for nonexpansive mappings. J. Math. Anal. Appl. 298, $279-291$ (2004)

6. Yao, Y, Noor, M: On viscosity iterative methods for variational inequalities. J. Math. Anal. Appl. 325, 776-787 (2007)

7. Yamada, I: The hybrid steepest-descent method for variational inequality problems over the intersection of the fixed-point sets of nonexpansive mappings. In: Inherently Parallel Algorithms in Feasibility and Optimization and Their Applications (Haifa, 2000). Studies in Computational Mathematics vol. 8, pp. 473-504 (2001)

8. Buong, D, Duong, LT: An explicit iterative algorithm for a class of variational inequalities in Hilbert spaces. J. Optim. Theory Appl. 151, 513-524 (2011)

9. Blum, E, Oettli, W: From optimization and variational inequalities to equilibrium problems. Math. Stud. 63, 123-145 (1994)

10. Tian, M, Di, LY: Synchronal algorithm and cyclic algorithm for fixed point problems and variational inequality problems in Hilbert spaces. Fixed Point Theory Appl. 2011, 21 (2011) 
11. Zhou, HY, Wang, P: A simpler explicit iterative algorithm for a class of variational inequalities in Hilbert spaces. J. Optim. Theory Appl. (2013). doi:10.1007/s10957-013-0470-x

12. López, G, Martin, V, Xu, HK: Iterative algorithm for the multi-sets split feasibility problem. In: Biomedical Mathematics: Promising Directions in Imaging, Therapy Planning and Inverse Problems, pp. 243-279 (2009)

13. Marino, G, Xu, HK: Weak and strong convergence theorems for strict pseudo-contractions in Hilbert spaces. J. Math Anal. Appl. 329, 336-346 (2007)

14. Suzuki, T: Strong convergence theorems for an infinite family of nonexpansive mappings in general Banach spaces. Fixed Point Theory Appl. 1, 103-123 (2005)

10.1186/1687-1812-2014-68

Cite this article as: Duan and He: Generalized viscosity approximation methods for nonexpansive mappings. Fixed Point Theory and Applications 2014, 2014:68

Submit your manuscript to a SpringerOpen ${ }^{\odot}$ journal and benefit from:

- Convenient online submission

- Rigorous peer review

- Immediate publication on acceptance

- Open access: articles freely available online

- High visibility within the field

- Retaining the copyright to your article

Submit your next manuscript at $>$ springeropen.com 